\title{
Integriertes Wassermanagement
}

\author{
Thomas Sterr
}

Online veröffentlicht: 26.11.2009

(C) Springer-Verlag 2009

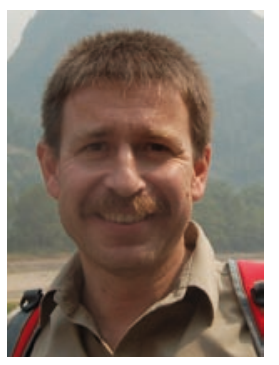

Integriertes Wassermanagement versucht, dem nachhaltigen Umgang mit der für alles Leben auf Erden zentralen Ressource Wasser auf Basis eines umfassenden Systemverständnisses gerecht zu werden. Der integrative Charakter entsprechender Ansätze reicht dabei von großräumlichen über regionale Ansätze (wie bspw. einem mannigfaltig integrierten Flusseinzugsgebietsmanagement) bis hin zu EDV-technischen Lösungen zur integrativen Steuerung und Optimierung einer innerbetrieblichen Wasserkreislaufwirtschaft. Auch rein technologisch beschriebene Lösungsstrategien sollten sich beim integrierten Wassermanagement als Resultate einer diskursiven Abstimmung mit aktiv Beteiligen und passiv Betroffenen darstellen, die gleichzeitig auch in das ökosphärische Umsystem nachhaltigkeitsorientiert eingebettet sind. Ebenso sind ökonomische, ökologische und soziale Aspekte eines integrierten Wassermanagements nicht nur im Rahmen kurzfristiger Betrachtungen miteinander in Beziehung zu setzen und abzustimmen, sondern darüber hinaus auch über einen Mechanismus

Dr. Thomas Sterr $(\varangle)$

IUWA - Institut für Umweltwirtschaftsanalysen Heidelberg e. V.,

Tiergartenstraße 17, 69121 Heidelberg, Deutschland

E-Mail: sterr@iuwa.de

des Backcasting ${ }^{1}$, durch den relevantes Wissen um mittel- und langfristigen Konsequenzen entsprechender Problemlösungsstrategien bereits in die Ausgestaltung heutiger Entscheidungen einbezogen werden kann. Im Sinne einer systemorientierten Herangehensweise bietet sich an, integriertes Wassermanagement als flexiblen Knoten-KantenGeflechts zu begreifen und auszugestalten, das einerseits inneren Entfaltungs- und Umgestaltungsmöglichkeiten hinreichend Raum gibt, andererseits aber auch auf Veränderungen der Systemumwelt mit innovativen Antworten im Sinne der Nachhaltigkeitsförderung zu reagieren versteht.

Dies alles klingt auf den ersten Blick extrem anspruchsvoll und dennoch steckt hinter dem Begriff des integrierten Wassermanagements kein holistischer Anspruch, der letztlich alles mit allem verknüpfen wollte. Schließlich impliziert ja bereits der Begriff „Management“ eine sachzielorientierte Planung, Durchführung, Kontrolle und Steuerung von Prozessen, wie sie nur durch einen Prozess der Selektion relevanter Systemgrößen erreicht werden kann. Die Kunst integrierter Wassermanagementansätze besteht deshalb darin, dass es gelingt, konkrete wissenschaftliche Erkenntnisse und umsetzungspraktische Kompetenzen unterschiedlichster Provenienz, bzw. Akteure unterschiedlicher Interessenlagen so miteinander zu verknüpfen, dass sie im Sinne einer nachhaltigkeitsorientierten Bewirtschaftung der Ressource Wasser fruchtbar zusammenwirken.

Die uwf-Schwerpunktausgabe „integriertes Wassermanagement" offeriert eine Reihe ausgewählter Anregungen und Fallbeispiele, denen neben einem entsprechenden Nachhaltigkeitsanspruch auch die Tatsache gemein ist, dass sie nicht nur beschreiben, sondern gerade auch ökonomische Begründungen zur Gangbarkeit bzw. Überlegenheit entsprechender Ausgestaltungsvorschläge anbieten.

\footnotetext{
${ }^{1}$ Siehe hierzu auch der Fachbeitrag von Nowack/Günther in diesem Heft.
} 
Der Reigen entsprechender Fachbeiträge beginnt mit einer Stellungnahme von Dr-Ing. Martin Grambow, der als Abteilungsleiter Wasserwirtschaft im Bayrischen Staatsministerium für Umwelt, Gesundheit und Verbraucherschutz Verantwortung trägt und erst im letzten Jahr (2008) ein viel beachtetes Buch zum „Integrierten Wasser-Ressourcenmanagement" veröffentlicht hat ${ }^{2}$. Im folgenden Beitrag thematisiert Grambow bei seinen Überlegungen zum „Integrierten Wasser-Ressourcenmanagement als Antwort auf drängende Wasserfragen" sowohl gesellschaftlich-politische, rechtliche, ökologische wie auch technisch-wirtschaftliche Aspekte. Gleichzeitig unternimmt er mit dem interessierten Leser eine teilweise sogar sehr persönlich gehaltene Reise zwischen globalem Verantwortungsbewusstsein und regionalen Umsetzungsoptionen. Auf diese Art und Weise begründet der Beitrag von Grambow gleich zu Beginn des Themenheftes einerseits die Notwendigkeit integrierter Wassermanagementansätze, gibt aber andererseits auch schon Hinweise darauf, wo bereits entsprechend zielführende Wege beschritten werden, bzw. wodurch ihre Verfolgung erleichtert werden könnte.

Mit Prof. Dr. Uwe Grünewald kommt im direkten Anschluss ein Wissenschaftler zu Wort, der sich als Lehrstuhlinhaber für Hydrologie und Wasserwirtschaft an der TU Cottbus bereits seit vielen Jahren mit entsprechenden Sachfragen beschäftigt und bei vielen seiner Forschungsprojekte insbesondere Fragen des Flussgebietsmanagements im Blick hat. Der Fachbeitrag von Grünewald fokussiert hierbei auf die hydrologischen Herausforderungen, die die extrem kurzfristige Schließung großräumiger Braunkohletagebaubetriebe in der Niederlausitz in den Jahren nach 1990 mit sich brachte und bis heute noch zeitigt. Tatsächlich waren die dortigen Entwicklungen in den letzten 20 Jahre begleitet von der Herausbildung vollkommen neuer Oberflächen- und Grundwasserlandschaften, die auch heute noch weit entfernt von einem dynamischen Systemgleichgewicht sind, weshalb unerwünschte Folgewirkungen gegenwärtiger Prozessabläufe durch umfangreiche Monitoringprozesse überwacht und über wohldosierte Systemeingriffe abgepuffert werden müssen.

Ebenfalls mit der Frage einer nachhaltigkeitsorientierten Bewirtschaftung von Gewässerlandschaften beschäftigt sich der Fachbeitrag von Prof. Dr. Hans-Ulrich Zabel, für diesen Fall allerdings mit einer Argumentationspalette, die dazu beitragen soll, folgenschwere Eingriffe in ein naturnahes Ökosystem zu verhindern. Als Lehrstuhlinhaber für Betriebswirtschaftslehre an der Martin-Luther Universität Halle-Wittenberg begründet Zabel seinen Feldzug gegen einen weitergehenden Elbausbau für die Binnenschifffahrt primär ökonomisch und trifft hierdurch die Protagonisten

\footnotetext{
${ }^{2}$ Grambow (2008) Wassermanagement - Integriertes Wasser-Ressourcenmanagement von der Theorie zur Umsetzung“"
}

entsprechender Investitionsmaßnahmen ausgerechnet in der Klasse der von jenen als besonders relevant dargestellten Entscheidungsparameter. Unabhängig von der rein sachlichen Fragestellung wird an diesem Aufsatz deutlich, dass auch die Betriebswirtschaftslehre heute mit einer ganzen Reihe ökonomischer Analyse- und Bewertungsinstrumente operiert, die im Werkzeugkasten für ein integriertes Wassermanagements hilfreiche Dienste leisten können.

Mit der für die Prognose zukünftiger Realitäten besonders hilfreichen Szenariotechnik, die in der betriebswirtschaftlichen Forschung und Anwendung heute ebenfalls eine wichtige Rolle spielt, beschäftigt sich der darauf folgende Artikel von Prof. Dr. Edeltraut Günther und ihrem Doktoranden Martin Nowack ${ }^{3}$ vom Lehrstuhl für Betriebswirtschaftslehre der TU Dresden. Die Beiden wenden das Verfahren in seiner besonderen Spezifikation als „Dresdner Szenariotechnik“ auf das Integrierte Wasserressourcenmanagement an und verdeutlichen dabei auch die funktionalen Vorzüge einer derartigen Szenarioplanung als Entscheidungsgrundlage. Anhand einer stichwortartigen Kurzbeschreibung zentraler Charakteristika wichtiger Szenariostudien im Wasserbereich geben sie dem interessierten Leser wichtige Hinweise für eine eingehendere Beschäftigung mit der Anwendung derartiger Verfahren.

Ebenfalls mit betriebswirtschaftlichen Argumenten begründen die Mitarbeiter des Ladenburger ABB Forschungszentrums Markus Gauder, Frederik Blank und Werner Schmidt ihren Aufbau eines integrierten Wassermanagements als „,... leitsystembasierter Ansatz zur Unterstützung des nachhaltigen Betriebs von Wasserversorgungsnetzen“. In ihren Ausführungen widmen sie sich dabei insbesondere der Problematik der Leitungsverluste, die gemäß der von ihnen zitierten VEWA Studie von 2006 selbst in europäischen Ländern zu Einbußen von 6-50\% des ins Netz eingespeisten Trinkwassers führen. Auf Basis einer entsprechenden Ursachenanalyse haben sie deshalb eine leitsystembasierte Systemarchitektur entwickelt, die Druckmanagement, Pumpeneinsatzplanung und weitere Komponenten eines aktiven Leckagemanagements EDV-technisch zu einer konsistenten Informationsbasis verbindet und so eine umfassende Entscheidungsgrundlage für das Monitoring sowie die gesamtsystemische Optimierung von Wasserverteilungsnetzen bietet.

Vor dem Hintergrund der Tatsache, dass allenfalls einzelne Großunternehmen von sich behaupten könnten, im Bereich „integriertes Wassermanagement“ tatsächlich als Systemanbieter auftreten zu können, kommt der im April 2008 gegründeten „German Water Partnership“ (GWP) hier eine besonders wertvolle Vernetzungsfunktion zu. Trotz ihrer jungen Entwicklungsgeschichte hat es die GWP unter Lei-

\footnotetext{
${ }^{3}$ Gegenwärtig durchgeführtes Dissertationsvorhaben: „Managing the Interface of Demographic Change on the Urban Drainage Organizations by Means of Scenario Planning“.
} 
tung ihres Geschäftsführers Stefan Girod bereits verstanden, auch auf bedeutenden internationalen Foren und Marktplätzen eindrücklich Flagge zu zeigen und die vielschichtige Problemlösungskompetenz ihrer inzwischen 230 Mitglieder zu demonstrieren. Mit ,adapted solutions for our partners abroad $^{\text {“4 }}$ sollen hierbei weltweit Ansätze zum integrierten Wassermanagement durch Konsortien hochspezialisierter Akteure der deutschen Wirtschaft und Wissenschaft gesamtsystemisch durchgeplant und weltweit umgesetzt werden. Entsprechende Ansätze exemplifiziert der Fachbeitrag von Girod und Bismuth anhand zweier aktuell in Umsetzung befindlicher Projekte in Vietnam (IWRM Vietnam) sowie der Mongolei (MoMo).

Ebenfalls mit integriertem Wassermanagement in einem asiatischen Kontextmilieu beschäftigt sich schließlich der Aufsatz von Dr. Klaus Baier, Avaro Real, Ramona Strohschön unter Leitung des Lehrstuhlinhabers für Ingenieurgeologie und Hydrogeologie der RWTH Aachen, Herrn Prof. Dr. Rafig Azzam. Am Beispiel der südchinesischen Millionenstadt Guangzhou verweisen sie auf die besondere Problematik urbaner Grundwasserneubildung, die sich in diesem Falle primär aus urbanen Abwässern speist und daher in ganz erheblichem Maße zur Grundwasserverschlechterung beiträgt. Abhilfe soll dabei durch dezentrale, an lokale Mikrostrukturen angepasste Wasserver- und -entsorgungslösungen geschaffen werden, wobei die Entfernung von Schadstoffen aus dem Grundwasser ganz konkret über den Einsatz elektrokinetischer Prozesse (EKP) getestet werden soll.

Ebenfalls mit einem chinesischen Anwendungskontext, gleichwohl allerdings unter semiariden Umfeldbedingungen, beschäftigt sich schließlich der Artikel zur Entwicklung eines integrierten Wassermanagements für das Industriegebiet Midong, das gegenwärtig als größte Flächeneinheit industriewirtschaftlicher Entwicklung im Nordwesten Chinas entwickelt wird. Die Autorin Katharina Fricke verdeutlicht in diesem Zusammenhang die zumindest latente Wasserknappheit sowie die mit der nicht nachhaltigen Grundwasserextraktion verbundene zunehmende Ausbeutung des Grundwasserkörpers. Hier wird es insbesondere darauf ankommen, dass Kommune, Haushalte, Landwirtschaft und Industrie zugunsten einer effizienteren Wassernutzung zukünftig enger und proaktiver zusammenarbeiten, wobei der Förderung eines ressourcenschonenden, kreislauforientierten Wassermanagements besondere Bedeutung beizumes-

\footnotetext{
${ }^{4}$ GWP (oJ), S. 7
}

sen ist. Dieser und andere Ansätze zum integrierten Wassermanagement wurden jüngst auch in einer vom BMBF finanzierten 56-seitigen Broschüre des UFZ beschrieben, die von Dr. Ralf Ibisch und Prof. Dr. Dietrich Borchardt zusammengestellt wurde ${ }^{5}$ und im Internet derzeit unter www. wasserressoucen-management.de kostenlos in deutscher und englischer Sprache heruntergeladen werden kann.

Als besonders innovativer Ansatz im Sinne eines zukunftsweisend integrierten Wassermanagements sei abschließend noch auf die Initiative „Öko-Bauern“ der Stadtwerke München (SWM) hingewiesen, mit der das Unternehmen bereits seit 1992 den ökologischen Landbau in seinem Trinkwassereinzugsgebiet Mangfalltal fördert, um hierdurch die qualitativen Eigenschaften seines hochsensiblen Produktes auch mittel- und langfristig abzusichern. Zur weiteren Stabilisierung bzw. Absicherung einer an natürlichen Kreislaufmechanismen orientierten Bodenbewirtschaftung unterstützen die SWM ihre Partner aus der Landwirtschaft dabei sogar bei der professionellen Vermarktung entsprechender Bioprodukte. Gemeinsam mit den Öko-Verbänden haben sie hierzu eine Arbeitsgemeinschaft ins Leben gerufen. „Wer sich gesund ernähren und gleichzeitig etwas für die Reinhaltung des Münchner Trinkwassers tun möchte, der sollte beim Einkauf auf Produkte aus dem Mangfalltal achten" ${ }^{\text {"6 }}$.

\section{Literatur}

Grambow M (2008) „Wassermanagement: Integriertes Wasser-Ressourcenmanagement von der Theorie zur Umsetzung“. Vieweg/ GWV Fachverlage; Wiesbaden

GWP (German Water Partnership) (oJ) Excellence in Water Technology \& Water Management. Booklet on Capacities, Organisational Structures and Actors

Stadtwerke München (Internet, 12.11.2009) „M-Wasser - ein erstklassiges Naturprodukt“. http://www.swm.de/dokumente/swm/ pdf/infomaterial/mwasser.pdf

UFZ (Umweltforschungszentrum) (2009) IWRM - Integrated Water Resources Management: From Research to Implementation. UFZ Leipzig/Magdeburg, August 2009-11-13

UFZ (Umweltforschungszentrum) (Internet, 12.11.2009) download: IWRM - Integriertes Wasserressourcen-Management: Von der Forschung zur Umsetzung http://www.wasserressourcen-management.de/_media/IWRM_Broschuere_Neuauflage_dt_Web.pdf

\footnotetext{
${ }^{5}$ UFZ (Umweltforschungszentrum) Leipzig: IWRM: Integrated Water Resources Management.

${ }^{6}$ Zitiert aus „Trinkwasserschutz durch ökologischen Landbau: ackern für die Umwelt“. S. 18-19 der SWM download-Broschüre: „M-Wasser - ein erstklassiges Naturprodukt“.
} 